\title{
Comparação entre os resultados pós-operatórios de pacientes submetidos ao procedimento tríplice e transplante de córnea combinado a fixação secundária de lente intra-ocular
}

\author{
Comparison between the postoperative results of triple procedure and \\ combined penetrating keratoplasty/transsclerally sutured posterior \\ chamberlensimplantation
}

\author{
Daniela Maggioni Pereira Leão ${ }^{1}$ \\ Luciene Barbasa de Sousa ${ }^{2}$
}

${ }^{1}$ Formada em oftalmologia pela Universidade de Alfenas. Estagiária do setor de Córnea e Doenças Externas do Hospital Oftalmológico de Sorocaba - Sorocaba (SP) Brasil.

${ }^{2}$ Doutora em Medicina pela UNIFESP - Chefe do Setor de Córnea e Doenças Externas do Departamento de Oftalmologia da Universidade Federal de São Paulo UNIFESP - São Paulo (SP) - Brasil. Coordenadora Responsável pela Residência Médica em Oftalmologia do Hospital Oftalmológico de Sorocaba - Sorocaba (SP) - Brasil.

Endereço para correspondência: Daniela Maggioni Pereira Leão. Rua Maria Lucinda, 455 - Apto. 61 Guarulhos (SP) CEP 07090-160

E-mail: dmaggioni@bol.com.br

Recebido para publicação em 11.11.2004

Versão revisada recebida em 14.10.2005

Aprovação em 16.05.2006

\begin{tabular}{|l|}
\hline \multicolumn{1}{|c|}{ RESUMO } \\
\hline Objetivo: Comparar os resultados pós-operatórios de 2 grupos de pa- \\
cientes submetidos a transplante de córnea com técnicas e tempo \\
cirúrgico diferentes, em relação à abordagem do cristalino e/ou lente \\
intra-ocular. Métodos: Neste estudo retrospectivo foram analisados 37 \\
olhos de pacientes divididos em 2 grupos: extração de catarata, implante \\
de lentes intra-oculares (LIO) e transplante de córnea no mesmo tempo \\
cirúrgico - grupo 1 (G1) extração de catarata sem implante de lentes intra- \\
oculares no primeiro tempo cirúrgico e fixação secundária de lentes intra- \\
oculares associada a transplante de córnea no segundo tempo cirúrgico \\
- grupo 2 (G2). As variáveis estudadas foram: acuidade visual, pressão \\
intra-ocular(PIO), astigmatismo refracional, astigmatismo ceratométrico \\
e complicações pós-operatórias. Resultados: Foi observado melhora da \\
acuidade visual nos 2 grupos (G1 p<0,001 e G2 p=0,008). O grupo 2 (G2) \\
apresentou pressão intra-ocular maior quando se comparou a pressão \\
intra-ocular pós-operatória entre os 2 grupos (p=0,014). Em relação aos \\
astigmatismos refrativo e ceratométrico não foi encontrada diferença \\
entre os grupos estudados, em cada variável. O tempo de seguimento \\
médio foi de 11 meses. Conclusão: Concluiu-se que o mais importante \\
fator negativo para a melhora da acuidade visual foi a variabilidade da \\
curvatura corneana, que tempos cirúrgicos diferentes podem levar à \\
perda da integridade do segmento anterior e posterior e piores resultados \\
da pressão intra-ocular, com desenvolvimento maior de glaucoma.
\end{tabular}

Descritores: Ceratoplastia penetrante; Extração de catarata; Implante de lente intraocular; Transplante de córnea; Complicações pós-operatórias; Estudo comparativo

\section{INTRODUÇÃO}

O transplante de córnea é um procedimento fascinante devido às inúmeras indicações para seu uso e aos métodos pelos quais pode ser combinado ${ }^{(1)}$.

A indicação mais freqüente para o transplante de córnea é a baixa de acuidade visual secundária a opacidade corneal. Outras indicações incluem irregularidade da superfície corneal, tratamento de afinamentos ou perfurações, alívio da dor, remoção de focos infecciosos e indicação cosmética ${ }^{(2)}$.

Cerca da metade dos transplantes penetrantes são combinados com 
498 Comparação entre os resultados pós-operatórios de pacientes submetidos ao procedimento tríplice e transplante de córnea combinado a fixação secundária de lente intra-ocular

uma variedade de outros procedimentos cirúrgicos. A cirurgia de catarata e de implante secundário de lente intra-ocular (LIO) são os mais comuns ${ }^{(1)}$.

As técnicas cirúrgicas para a reabilitação dos pacientes com doença corneal, em associação a catarata, têm mudado drasticamente nos últimos anos. Cada avanço na técnica cirúrgica de extração da catarata tem sido adaptada pelos cirurgiões de córnea nos procedimentos combinados com transplante, reduzindo a incidência de descolamento de retina e glaucoma do afácico e pseudo-fácico ${ }^{(3)}$.

Quanto ao implante secundário de lente intra-ocular, o posicionamento destas lentes na ausência de suporte capsular adequado é um desafio ${ }^{(4)}$. Os "designs" de algumas LIOs podem predispor à perda endotelial progressivamente maior de células endoteliais, como as LIOs de câmara anterior ${ }^{(5)}$. A descompensação corneal em presença destas lentes é uma das principais indicações para transplante de córnea com remoção da mesma ${ }^{(6)}$.

As LIOs de câmara posterior, em contraste, têm se mostrado adequadas para o uso juntamente com o transplante, pois, além de promover boa visão, estão relacionadas a um pequeno risco de descompensação endotelial do botão corneal ${ }^{(5)}$ por apresentar uma localização anatomicamente similar a do cristalino ${ }^{(6)}$.

Este estudo tem a finalidade de comparar os resultados pós-operatórios de 2 grupos de pacientes submetidos a transplante de córnea com técnicas e tempos cirúrgicos diferentes, em relação à abordagem do cristalino e/ou da LIO, ressaltando vantagens e desvantagens entre as técnicas.

\section{MÉTODOS}

Neste trabalho foram analisados os prontuários de 37 pacientes do Hospital Oftalmológico de Sorocaba, na cidade de Sorocaba - SP, sendo 26 pacientes do sexo feminino e 11 do sexo masculino. A idade média destes pacientes foi de 63,6 anos, variando de 40 a 88 anos e foram analisados 14 OD e 21 OE. As pesquisas se concentraram no período de janeiro de 1999 a janeiro de 2002. Estes pacientes foram divididos em 2 grupos, sendo 23 pacientes no grupo 1, 14 no grupo 2 . No Grupo 1 (G1) os pacientes foram submetidos à extração da catarata, implante de LIO de câmara posterior (CP) e transplante de córnea no mesmo tempo cirúrgico e no Grupo 2 (G2) a

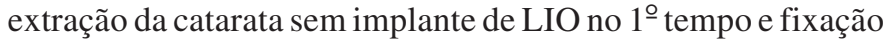
secundária de LIO de câmara posterior e transplante de córnea no $2^{\circ}$ tempo cirúrgico. Todos os pacientes apresentavam acuidade visual $\leq 0,4$ antes da cirurgia. As variáveis estudadas foram: acuidade visual, pressão intra-ocular, astigmatismo refracional e ceratométrico e complicações pós-operatórias. A fórmula utilizada para o poder da LIO foi a SRK/T na maioria dos casos, sendo a Holladay utilizada em 5 casos. A constante utilizada para os procedimentos tríplices variou entre 118,3 e 118,7, já para os implantes secundários de LIOs a constante usada foi 118,7. A ceratometria média foi baseada na ceratometria de cada paciente.

Foi aplicada a conversão para $\log$ MAR dos valores da acuidade visual em decimais e os valores transformados foram analisados. Desta análise foram excluídos 4 pacientes por apresentarem acuidade visual (AV) igual a percepção luminosa (PL) na avaliação pré ou na pós-operatória ${ }^{(7)}$.

As patologias pré-operatórias dos pacientes de ambos os grupos estão descritas na tabela 1 , juntamente com a descrição de outros dados estudados.

Todos os procedimentos cirúrgicos foram realizados com anestesia peribulbar e em todos os casos utilizou-se o anel de Flieringa para sustentação do segmento anterior. As cirurgias foram realizadas por diferentes cirurgiões ainda em treinamento (residentes de terceiro ano e estagiários).

\section{Procedimento tríplice}

A técnica cirúrgica para o transplante de córnea envolveu uma trepanação corneana com diferença entre o botão doador e receptor de 0,25 ou $0,50 \mathrm{~mm}$.

Após a trepanação foi realizada capsulotomia anterior, luxação do núcleo, irrigação e aspiração de massas e colocado viscoelástico no saco capsular para a introdução da LIO de $\mathrm{CP}$, previamente calculada.

Quando houve necessidade, foi colocado carbacol intraocular para fechamento pupilar.

A câmara anterior foi inicialmente refeita com viscoelástico, o botão corneano foi suturado com nylon 10.0 e pontos separados, num total de 16 pontos. À medida que a câmara anterior foi se tornando mais estável o viscoelástico foi sendo substituída por solução salina balanceada.

\section{Procedimento combinado (transplante de córnea e implante secundário de LIO)}

Neste procedimento cirúrgico os pacientes já haviam sido submetidos à extração de catarata por facectomia ou facoemulsificação sem implante de LIO.

\begin{tabular}{|c|c|c|}
\hline Parâmetros & $\begin{array}{c}\mathrm{Tx}+\underset{(n=23)}{\mathrm{FEC}} \mathrm{cl} \text { LIO } \\
(\mathrm{n}=2\end{array}$ & $\begin{array}{c}\text { Tx + fixação } \\
(n=14)\end{array}$ \\
\hline \multicolumn{3}{|l|}{ Sexo - n (\%) } \\
\hline Feminino & $18(78,3)$ & $8(57,1)$ \\
\hline Masculino & $5(21,7)$ & $6(42,9)$ \\
\hline \multicolumn{3}{|l|}{ Idade (anos) } \\
\hline Média \pm dp & $65,0 \pm 9,0$ & $63,7 \pm 14,8$ \\
\hline Mínimo - máximo & $46-83$ & $40-88$ \\
\hline \multicolumn{3}{|l|}{ Olho - n (\%) } \\
\hline Direito & $10(43,5)$ & $6(42,9)$ \\
\hline Esquerdo & $13(56,5)$ & $8(57,1)$ \\
\hline \multicolumn{3}{|l|}{ Diagnóstico pré - n (\%) } \\
\hline $\begin{array}{l}\text { Ceratopatia bolhosa } \\
\text { pseudo-fácica }\end{array}$ & & $12(85,7)$ \\
\hline Distrofia de Fuchs & $11(47,8)$ & $1(7,1)$ \\
\hline Rejeição & $1(4,3)$ & \\
\hline Astigmatismo irregular alto & $2(8,7)$ & \\
\hline Leucoma & $9(39,1)$ & $1(7,1)$ \\
\hline
\end{tabular}


Inicialmente, dois "flaps" esclerais externos, nos meridianos das $3 \mathrm{~h} \mathrm{e} 9 \mathrm{~h}$, foram efetuados a $1 \mathrm{~mm}$ do limbo aproximadamente. Similar à técnica anterior, foi trepanado a córnea receptora com um trépano 0,25 ou $0,50 \mathrm{~mm}$ menor que a córnea doadora. Feito vitrectomia anterior no plano pupilar e em toda margem pupilar da íris, a LIO de câmara posterior foi suturada ao fio de polypropyleno 10.0, biagulhado, um nó de cada alça da LIO, utilizando os orifícios existentes nela. Foi então, implantada a LIO dentro do sulco ciliar sendo suturada às $3 \mathrm{~h} e$ $9 \mathrm{~h}$, nos respectivos "flaps" esclerais. O viscoelástico foi colocado sobre a LIO e superfície da íris e o botão doador foi suturado com nylon 10.0, pontos separados (16 pontos). Posteriormente, o viscoelástico foi substituído por solução salina balanceada. Os "flaps" esclerais foram suturados também com nylon 10.0 e cobertos pela conjuntiva.

Os três procedimentos acima receberam injeção subconjuntival de dexametasona e gentamicina.

No pós-operatório todos os pacientes receberam acetato de prednisolona $1 \%$ tópica em esquema de regressão, por no mínimo 6 meses e o floxacina $0,3 \%$ tópica, 4 vezes ao dia, por aproximadamente 15 dias ou até o fechamento completo do epitélio corneal. As avaliações foram mensais com medição da PIO e exame na lâmpada de fenda. A melhor acuidade visual com correção só se obteve após a remoção de todos os pontos necessários para diminuição do astigmatismo refracional póscirúrgico, juntamente com a ceratometria final.

Nos grupos 2 o intervalo entre a extração de catarata sem implante de LIO de $\mathrm{CP}$ e o transplante de córnea com implante secundário de LIO de $\mathrm{CP}$ variou de 3 a 10 anos. Durante os exames pré-operatórios foi realizado ultra-sonografia nos pacientes cujo exame de fundo de olho era impossível devido opacidade da córnea com a finalidade de se pesquisar alterações em segmento posterior como descolamento de retina e principalmente edema macular cistóide (EMC). Na coleta de dados não havia descrição ultra-sonográfica destas patologias nos pacientes que foram submetidos aos procedimentos. $\mathrm{O}$ tempo de seguimento do G1 de 10 meses e do G2 de 12 meses.

\section{RESULTADOS}

Variáveis estudadas:

\section{Acuidade visual}

$\mathrm{Na}$ avaliação de AV pré-operatória, foi encontrada diferença estatisticamente significante entre os grupos submetidos aos dois tipos de cirurgia $(\mathrm{p}=0,012)$, onde o grupo submetido à Tx + Fixação apresentou AV pré-operatória em logMAR significantemente diferente do grupo Tx + FEC com LIO.

Em função dessa diferença pré, os grupos foram comparados em relação à variação entre as avaliações e nessa comparação não foi encontrada diferença estatisticamente significante entre os grupos $(\mathrm{p}=0,386)$, indicando que as variações apresentadas pelos 2 grupos foram semelhantes (Tabela 2 e Gráfico 1).
Quando comparadas às avaliações pré e pós dentro de cada grupo, os resultados foram:

- no grupo Tx + FEC com LIO foi encontrada variação estatisticamente significante entre as avaliações $(p<0,001)$, indicando uma melhora significante da $\mathrm{AV}$ no pós quando comparado ao pré.

- no grupo Tx + Fixação foi encontrada variação estatisticamente significante entre as avaliações $(\mathrm{p}=0,008)$, indicando uma melhora significante da AV no pós quando comparado ao pré.

\begin{tabular}{|c|c|c|}
\hline \multirow[t]{2}{*}{ AV em LogMAR } & \multicolumn{2}{|c|}{ Cirurgia } \\
\hline & $\begin{array}{c}\text { Tx }+\underset{(n=21)}{\text { FEC cl LIO }} \\
(n=210\end{array}$ & $\begin{array}{c}\text { Tx + fixação } \\
(n=12)\end{array}$ \\
\hline \multicolumn{3}{|l|}{ Pré } \\
\hline Média \pm dp & $2,02 \pm 0,77$ & $2,67 \pm 0,49$ \\
\hline Mínimo - máximo & $0,30-3,00$ & $2,00-3,00$ \\
\hline Mediana & 2,00 & 3,00 \\
\hline \multicolumn{3}{|l|}{ Pós } \\
\hline Média \pm dp & $0,15 \pm 0,17$ & $1,33 \pm 0,94$ \\
\hline Mínimo - máximo & $0,00-0,70$ & $0,22-3,00$ \\
\hline Mediana & 0,15 & 1,00 \\
\hline \multicolumn{3}{|l|}{ Variação (Pré-Pós) } \\
\hline Média \pm dp & $1,87 \pm 0,77$ & $1,34 \pm 1,11$ \\
\hline Mínimo - máximo & $0,18-2,95$ & $-1,00-2,48$ \\
\hline Mediana & 1,85 & 1,89 \\
\hline \multicolumn{3}{|c|}{$\begin{array}{l}\mathrm{n}=\text { número; } \mathrm{AV}=\text { acuidade visual; } \mathrm{dp}=\text { desvio padrão; } \text { LogMAR= Fórmula para } \\
\text { a conversão da } \mathrm{AV} \text { em decimais; } \mathrm{Tx}+\mathrm{FEC} \mathrm{c} / \mathrm{LIO}=\text { Transplante de córnea e } \\
\text { facectomia com implante de LIO; } T x+\text { fixação= Transplante de córnea e } \\
\text { fixação de LIO. } \\
\text { Prova de Kruskal-Wallis. }\end{array}$} \\
\hline
\end{tabular}

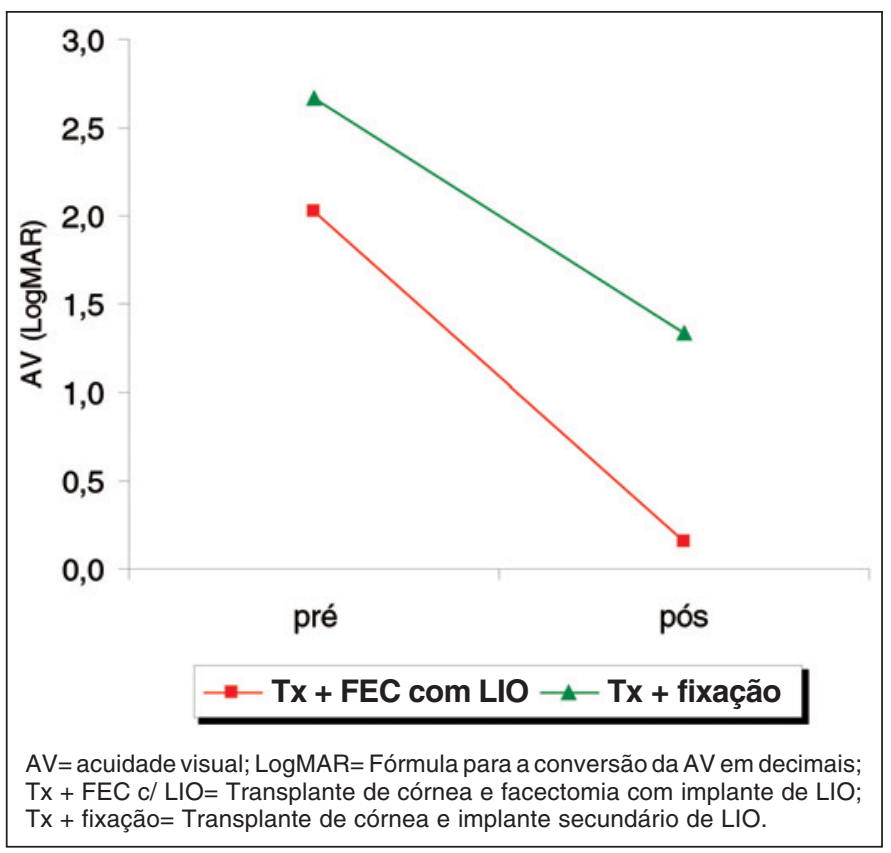

Gráfico 1 - Acuidade visual pré e pós-operatória por grupo estudado 
500 Comparação entre os resultados pós-operatórios de pacientes submetidos ao procedimento tríplice e transplante de córnea combinado a fixação secundária de lente intra-ocular

\section{Pressão intra-ocular}

Na avaliação da PIO pré-operatória, não foi encontrada diferença estatisticamente significante entre os grupos submetidos aos dois tipos de cirurgia $(\mathrm{p}=0,759)$.

Quando comparados em relação a PIO na avaliação pós, foi encontrada diferença estatisticamente significante entre os grupos $(\mathrm{p}=0,014)$, onde o grupo submetido à $\mathrm{Tx}+$ Fixação apresentou PIO pós-operatória significantemente maior do que a dor grupo Tx + FEC c/ LIO.

Não foi encontrada diferença estatisticamente significante entre os grupos em relação à variação entre as avaliações $(\mathrm{p}=0,345)$ (Tabela 3 e Gráfico 2).

Quando comparadas as avaliações pré e pós dentro de cada grupo, os resultados foram:

- no grupo Tx + FEC com LIO não foi encontrada variação estatisticamente significante entre as avaliações $(p=0,208)$.

- no grupo Tx + Fixação não foi encontrada variação estatisticamente significante entre as avaliações $(\mathrm{p}=0,142)$.

\section{Astigmatismo refracional}

Na avaliação do astigmatismo refracional na avaliação pós, não foi encontrada diferença estatisticamente significante entre os grupos $(\mathrm{p}=0,067)($ Tabela 4$)$.

\section{Astigmatismo ceratométrico}

O teste de Kolmogorov Smirnov foi aplicado para testar a hipótese de distribuição normal nas condições de avaliação e essa hipótese foi rejeitada em todas elas $(\mathrm{p}=0,025$ no pré do grupo Tx + FEC com LIO, $\mathrm{p}=0,002$ no pós do grupo Tx + FEC com LIO, $\mathrm{p}=0,036$ no pré do grupo Tx + Implante $2^{\circ}$ LIO e $\mathrm{p}=0,047$ no pós do grupo $\mathrm{Tx}+$ Implante $2^{\circ} \mathrm{LIO}$ ) e por esse motivo foram utilizados testes não paramétricos.

$\mathrm{Na}$ amostra de 37 pacientes avaliados quanto ao astigma-

\begin{tabular}{|c|c|c|}
\hline \multirow[t]{2}{*}{ PIO } & \multicolumn{2}{|c|}{ Cirurgia } \\
\hline & $\begin{array}{c}\mathrm{Tx}+\mathrm{FEC} \mathrm{c/} \mathrm{LIO} \\
(n=23)\end{array}$ & $\begin{array}{c}\text { Tx + fixação } \\
(n=14)\end{array}$ \\
\hline \multicolumn{3}{|l|}{ Pré } \\
\hline Média \pm dp & $12,70 \pm 3,21$ & $13,79 \pm 5,38$ \\
\hline Mínimo - máximo & $8-20$ & $8-28$ \\
\hline Mediana & 12 & 12 \\
\hline \multicolumn{3}{|l|}{ Pós } \\
\hline Média \pm dp & $13,83 \pm 2,85$ & $18,86 \pm 8,30$ \\
\hline Mínimo - máximo & $10-22$ & $10-40$ \\
\hline Mediana & 13 & 17 \\
\hline \multicolumn{3}{|l|}{ Variação (Pós-Pré) } \\
\hline Média \pm dp & $1,13 \pm 4,16$ & $5,07 \pm 11,17$ \\
\hline Mínimo - máximo & $-6-12$ & $-10-30$ \\
\hline Mediana & 0,00 & 2,50 \\
\hline \multicolumn{3}{|c|}{$\begin{array}{l}\mathrm{n}=\text { número; dp= desvio padrão; } \mathrm{PIO}=\text { pressão intra-ocular; } \mathrm{Tx}+\mathrm{FEC} \mathrm{c} / \mathrm{LIO}= \\
\text { Transplante de córnea e facectomia com implante de LIO; Tx + fixação= } \\
\text { Transplante de córnea e fixação de LIO. } \\
\text { Prova de Kruskal-Wallis. }\end{array}$} \\
\hline
\end{tabular}

tismo ceratométrico pré-operatório, não foi encontrada diferença estatisticamente significante entre os grupos submetidos aos dois tipos de cirurgia ( $\mathrm{p}=0,893)$.

Quando comparados em relação ao astigmatismo ceratométrico na avaliação pós, não foi encontrada diferença estatisticamente significante entre os grupos $(\mathrm{p}=0,171)$. Também não foi encontrada diferença estatisticamente significante entre os grupos em relação à variação entre as avaliações $(\mathrm{p}=0,116)$ pré e pós-operatórias (Tabela 5 e Gráfico 3).

Quando comparadas as avaliações pré e pós dentro de cada grupo, os resultados foram:

- no grupo Tx + FEC c/ LIO foi encontrada variação estatisticamente significante entre as avaliações $(p=0,048)$, indicando um aumento significante do astigmatismo no pós quando comparado ao pré;

- no grupo Tx + Implante $2^{\circ}$ LIO foi encontrada variação estatisticamente significante entre as avaliações $(p=0,003)$, indicando um aumento significante do astigmatismo no pós quando comparado ao pré.

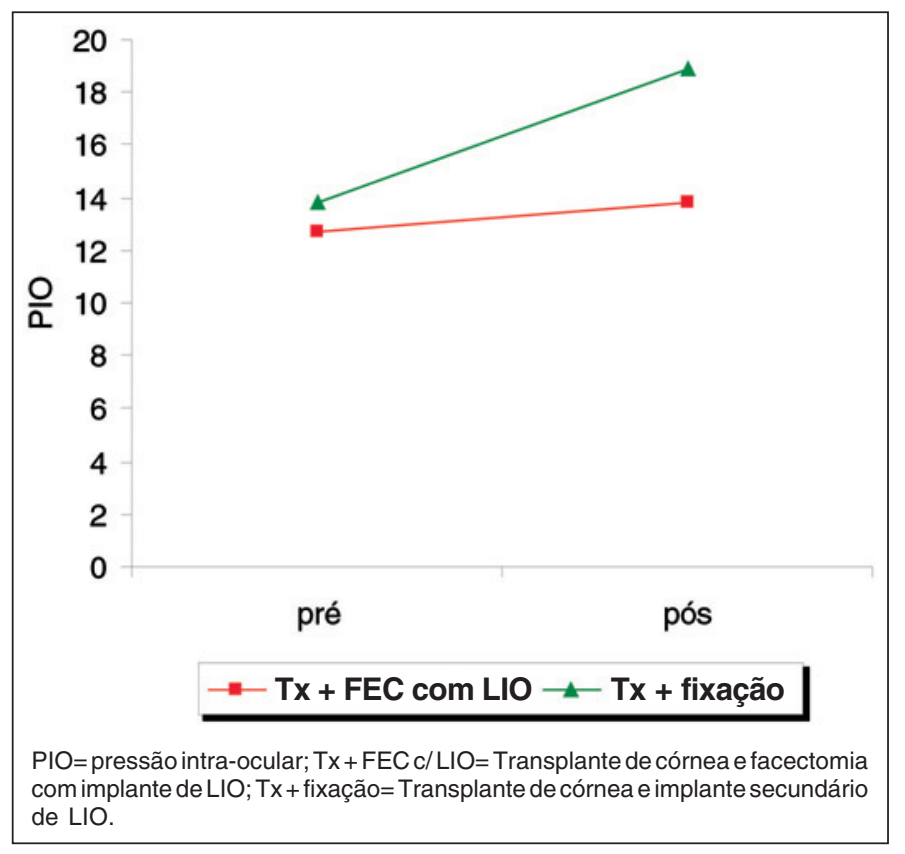

Gráfico 2 - Análise da variação da PIO pré e pós-operatórios em cada grupo

\begin{tabular}{|c|c|c|}
\hline \multirow{2}{*}{$\begin{array}{l}\text { Astigmatismo } \\
\text { refracional pós }\end{array}$} & \multicolumn{2}{|c|}{ Cirurgia } \\
\hline & $\begin{array}{c}\mathrm{Tx}+\underset{(n=23)}{\mathrm{FEC} \text { cl LIO }} \\
(\mathrm{n}=23\end{array}$ & $\begin{array}{c}\text { Tx + fixação } \\
(n=14)\end{array}$ \\
\hline Média $\pm d p$ & $2,22 \pm 2,16$ & $1,07 \pm 1,59$ \\
\hline Mínimo - máximo & $0,0-7,5$ & $0,0-5,0$ \\
\hline Mediana & 1,5 & 0,0 \\
\hline
\end{tabular}




\section{Complicações}

Foi encontrada diferença estatisticamente significante entre os grupos quanto à ocorrência de complicações $(\mathrm{p}=0,006)$, onde o grupo Tx + Fixação apresentou proporção de olhos com complicações $(57,1 \%)$ significantemente maiores do que a do grupo Tx + FEC com LIO $(17,4 \%)$ (Tabela 6).

As complicações em cada grupo foram:

- no grupo Tx + FEC com LIO ocorreram $3(13,0 \%)$ casos de rejeição e $1(4,3 \%)$ de glaucoma.

- no grupo Tx + Fixação ocorreram $1(7,1 \%)$ casos de rejeição, $7(50,0 \%)$ de glaucoma.

\begin{tabular}{|c|c|c|c|}
\hline \multirow{2}{*}{$\begin{array}{l}\text { Astigmatismo } \\
\text { ceratométrico }\end{array}$} & \multicolumn{2}{|c|}{ Cirurgia } & \multirow{2}{*}{$\begin{array}{l}\text { Prova de } \\
\text { Mann- } \\
\text { Whitney }\end{array}$} \\
\hline & $\begin{array}{c}\text { Tx + FEC c/ LIO } \\
(n=23)\end{array}$ & $\begin{array}{c}\text { Tx + fixação } \\
(n=14)\end{array}$ & \\
\hline \multicolumn{4}{|l|}{ Pré } \\
\hline Média $\pm d p$ & $2,26 \pm 2,82$ & $2,10 \pm 3,20$ & \multirow{3}{*}{$p=0,893$} \\
\hline Mínimo - máximo & $0,00-9,50$ & $0,00-12,25$ & \\
\hline Mediana & 1,32 & 1,00 & \\
\hline \multicolumn{4}{|l|}{ Pós } \\
\hline Média \pm dp & $4,20 \pm 2,90$ & $6,71 \pm 5,72$ & \multirow{3}{*}{$p=0,171$} \\
\hline Mínimo - máximo & $0,30-12,21$ & $0,00-22,38$ & \\
\hline Mediana & 3,79 & 4,68 & \\
\hline \multicolumn{4}{|l|}{ Variação (Pós-Pré) } \\
\hline Média \pm dp & $1,94 \pm 3,94$ & $4,61 \pm 5,56$ & \multirow{3}{*}{$p=0,116$} \\
\hline Mínimo - máximo & $-4,86-12,21$ & $-0,75-21,63$ & \\
\hline Mediana & 1,41 & 3,66 & \\
\hline \multicolumn{4}{|c|}{$\begin{array}{l}\mathrm{n}=\text { número; } \mathrm{dp}=\text { desvio padrão; Tx }+\mathrm{FEC} \mathrm{c} / \mathrm{LIO}=\text { Transplante de córnea e } \\
\text { facectomia com implante de LIO; Tx + fixação= Transplante de córnea e } \\
\text { fixação de LIO. } \\
\text { Prova de Mann-Whitney. }\end{array}$} \\
\hline
\end{tabular}

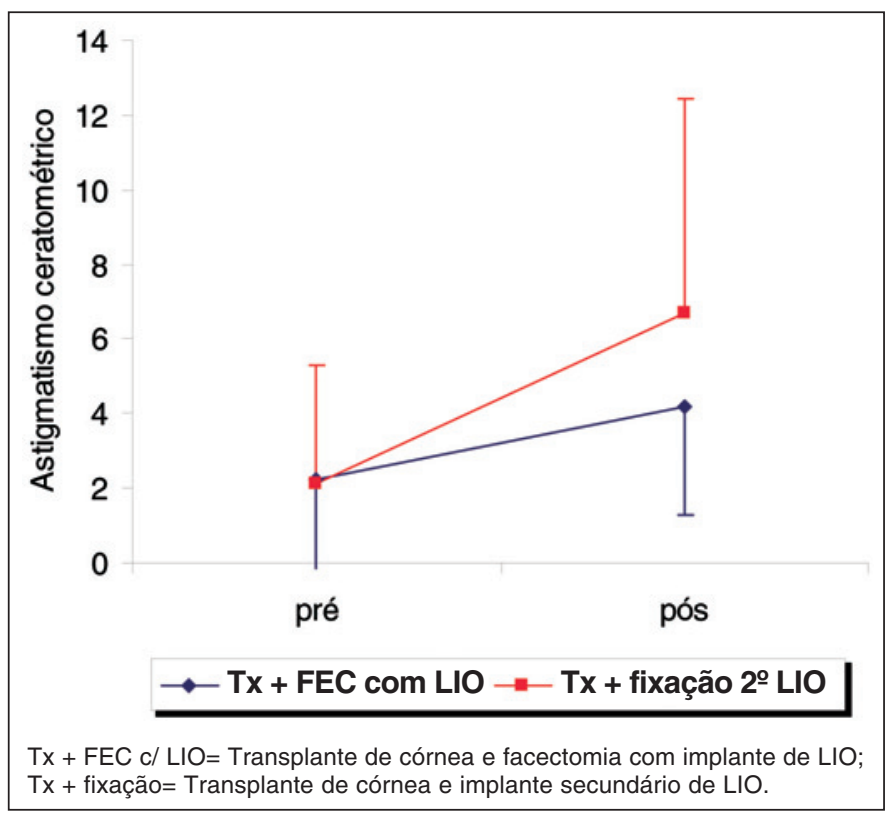

Gráfico 3 - Variação pré e pós-operatória do astigmatismo ceratométrico

\section{DISCUSSÃO}

As técnicas cirúrgicas para a reabilitação visual de pacientes que necessitam de transplante de córnea têm avançado muito nos últimos anos, a tal ponto que os cirurgiões de hoje se preocupam não só com a sobrevida do botão corneal como também com o erro refrativo dos pacientes no pós-operatório ${ }^{(8)}$.

Muitos estudos têm enfatizado que o procedimento tríplice com implante de LIO de CP se apresenta seguro e benéfico, já que a presença da LIO não prejudica a sobrevivência endotelial por manter a claridade do botão corneal ${ }^{(3)}$. Esses autores relataram que uma das maiores desvantagens é a incapacidade de prever o resultado refrativo pós-operatório pelo poder da LIO, com segurança, pois a curvatura corneal não pode ser determinada pré-operatoriamente ${ }^{(8)}$.

Ali et al. relataram que na literatura há relatos de transplantes associados à fixação secundária de LIO de CP com sutura transescleral, demonstrando um resultado favorável quanto à acuidade visual e aceitável taxa de complicações. No entanto a previsão do resultado refrativo pelo poder da LIO pode ser mais difícil, se comparado com a previsão para a implantação da LIO de CP no saco capsular, devido à localização final na fixação secundária ser menos precisa ${ }^{(4)}$.

Neste estudo houve uma variação estatisticamente significante do astigmatismo ceratométrico em cada grupo quando se comparou os resultados pré com os pós-operatórios, existindo um aumento deste astigmatismo ceratométrico pós-operatório que se justifica pela variabilidade da curvatura corneal depois do transplante.

Já quando comparados os astigmatismos ceratométrico pósoperatórios entre os dois grupos, não houve diferença significante demonstrando que as técnicas de posicionamento das LIOs nas cirurgias não influenciaram os resultados.

Embora os casos tenham apresentado valores de astigmatismo ceratométrico com ampla variação entre o pré e o pósoperatórios de cada grupo, não houve influência nos resultados refrativos, já que não existiu esta diferença em tal variável.

A média do astigmatismo refracional no grupo 1 foi de 2,22 dioptrias, mantendo-se como mostrado na maioria dos trabalhos, ou seja, de 2,00 dioptrias ${ }^{(1,3,8)}$. Já no grupo 2 o astigmatismo refracional foi de 1,07 dioptrias, permanecendo levemente abaixo da média dos estudos ${ }^{(1,3-4,8-9)}$.

\begin{tabular}{|c|c|c|c|}
\hline \multirow[t]{2}{*}{ Complicações } & \multicolumn{2}{|c|}{ Cirurgia } & \multirow{2}{*}{$\begin{array}{l}\text { Total } \\
\text { n (\%) }\end{array}$} \\
\hline & $\begin{array}{c}\text { Tx + FEC c/ LIO } \\
\text { n (\%) }\end{array}$ & $\begin{array}{c}\text { Tx + fixação } \\
n(\%)\end{array}$ & \\
\hline Ausente & $19(82,6)$ & $6(42,9)$ & $25(67,6)$ \\
\hline Presente & $4(17,4)$ & $8(57,1)$ & $12(32,4)$ \\
\hline Total & $23(100,0)$ & $14(100,0)$ & $37(100,0)$ \\
\hline
\end{tabular}


502 Comparação entre os resultados pós-operatórios de pacientes submetidos ao procedimento tríplice e transplante de córnea combinado a fixação secundária de lente intra-ocular

Quanto à acuidade visual, ambos os grupos tiveram melhora estatisticamente significante entre as avaliações pré e pós-operatórias, dentro de cada grupo.

Existiu diferença estatisticamente significante entre os grupos quando se comparou a PIO no pós-operatório, sendo encontrada uma PIO maior no grupo 2. Apesar de realizada ampla vitrectomia anterior e de terem sido utilizadas as LIOs de CP por agredirem menos o trabeculado devido à localização mais anatômica das mesmas ${ }^{(6)}$, estes pacientes possivelmente apresentavam dano na malha trabecular e/ou formação de sinéquia anterior prévia, pois já haviam sido submetidos à extração de catarata com ruptura de cápsula posterior e provável perda vítrea.

Este resultado justifica a ocorrência de $50 \%$ de glaucoma no grupo 2 quando analisadas as taxas de complicações entre os grupos.

\section{CONCLUSÃO}

Neste estudo comparou-se os resultados das cirurgias tríplice aos resultados dos transplantes de córnea com fixação secundária de LIO na sutura transescleral e ambos os procedimentos foram aceitáveis, sugerindo que podem ser realizados efetivamente sem nenhum dano ao paciente. Lembrando-se sempre que o maior fator negativo para a melhora da visão foi à variabilidade da curvatura corneal no pós-operatório e que pode haver aumento da PIO nos casos em que o paciente já foi submetido a procedimento cirúrgico prévio no olho a ser operado.

\section{ABSTRACT}

Purpose: To compare the outcomes of two surgical techniques of penetrating keratoplasty with different surgical time, regarding the crystalline and the intraocular lens. Methods: This retrospective study included 37 patients' eyes divided into 2 groups: extracapsular cataract extraction, posterior chamber intraocular lens implantation and penetrating keratoplasty (Group 1, G1) and transscleral fixation of posterior chamber lens and penetrating keratoplasty (Group 2, G2). The following parameters were recorded: visual acuity, intraocular pressure, refractive astigmatism, complication and keratometric astigmatism. Results: Visual acuity improved in the two groups $(\mathrm{G} 1 \mathrm{p}<0.001$ and $\mathrm{G} 2 \mathrm{p}=0.008)$. In $\mathrm{G} 2$ a significant change for the worse of intraocular pressure outcome was observed when compared with the other group $(\mathrm{p}=0.014)$. Regarding refractive and keratometric astigmatism no significant differences between the groups were found. The follow-up was 11 months. Conclusion: The most important negative prognostic factor affecting visual acuity was the postkeratoplasty corneal curvature which can not be predicted accurately and the most important negative factor affecting intraocular pressure was the loss of integrity of the anterior and posterior segments.

Keywords: Keratoplasty, penetrating; Cataract extraction; Lens implantation, intraocular; Corneal transplantation; Postoperative complications; Comparative study

\section{REFERÊNCIAS}

1. Krachmer JH, Mannis MJ, Holland EJ. Córnea. Fundamentals of cornea and external disease [CD-ROM]. St. Louis: Mosby; 1998.

2. Wilhelmus KR, Huang AJW, Hwang DG, Parrish CM, Sutphin JE, Whitsett JC. External disease and cornea. In: Liesegang TJ, Deutsch TA, Grand MG. Basic and clinical science course of ophthalmologists. San Francisco: The Foundation of the American Academy of Ophthalmology; 2001.

3. Kirkness CM, Cheong PY, Steele AD. Penetrating keratoplasty and cataract surgery: the advantages of an extracapsular technique combined with posterior chamber intraocular implantation. Eye. 1987;1(Pt 5):557-61.

4. Djalilian AR, George JE, Doughman DJ, Holland EJ. Comparison between the refractive results of combined penetrating keratoplasty/transsclerally sutured posterior chamber lens implantation and the triple procedure. Cornea. 1997;16 (3):319-21.

5. Chu MW, Font RL, Koch DD. Visual results and complications following posterior iris-fixated posterior chamber lenses at penetrating keratoplasty. Ophthalmic Surg. 1992;23(9):608-13.

6. Holland EJ, Daya SM, Evangelista A, Ketcham JM, Lubniewski AJ, Doughman DJ, et al. Penetrating keratoplasty and transscleral fixation of posterior chamber lens. Am J Ophthalmol. 1992;114(2):182-7.

7. Geggel HS. Intraocular lens implantation after penetrating keratoplasty. Improved unaided visual acuity, astigmatism, and safety in patients with combined corneal disease and cataract. Ophthalmology. 1990;97(11):1460-7.

8. Davis RM, Best D, Gilbert GE. Comparison of intraocular lens fixation techniques performed during penetrating keratoplasty. Am J Ophthalmol. 1991;111 (6):743-9.

9. Holladay JT. Proper method for calculating average visual acuity. J Refract Surg. 1997;13(4):388-91.

\section{Ao enviar um artigio para publicação, leia ATENTAMENTE as instruções para autores, constante no final de cada fascículo.}

